\title{
Long-term impact of the expansion of a hospital liaison psychiatry service on patient care and costs following emergency department attendances for self-harm
}

Joni Jackson*, Manjula D. Nugawela*, Frank De Vocht, Paul Moran, William Hollingworth, Duleeka Knipe, Nik Munien, David Gunnell and Maria Theresa Redaniel

\section{Background}

In September 2014, as part of a national initiative to increase access to liaison psychiatry services, the liaison psychiatry services at Bristol Royal Infirmary received new investment of f250 000 per annum, expanding its availability from 40 to $98 \mathrm{~h} \mathrm{per}$ week. The long-term impact on patient outcomes and costs, of patients presenting to the emergency department with selfharm, is unknown.

\section{Aims}

To assess the long-term impact of the investment on patient care outcomes and costs, of patients presenting to the emergency department with self-harm.

\section{Method}

Monthly data for all self-harm emergency department attendances between 1 September 2011 and 30 September 2017 was modelled using Bayesian structural time series to estimate expected outcomes in the absence of expanded operating hours (the counterfactual). The difference between the observed and expected trends for each outcome were interpreted as the effects of the investment.

\section{Results}

Over the 3 years after service expansion, the mean number of self-harm attendances increased $13 \%$. Median waiting time from arrival to psychosocial assessment was $2 \mathrm{~h}$ shorter (18.6\% decrease, $95 \%$ Bayesian credible interval (BCl) $-30.2 \%$ to $-2.8 \%$, there were 45 more referrals to other agencies $(86.1 \%$ increase, $95 \% \mathrm{BCl} 60.6 \%$ to $110.9 \%$ ) and a small increase in the number of psychosocial assessments $(11.7 \%$ increase, $95 \% \mathrm{BCl}-3.4 \%$ to $28.5 \%)$ per month. Monthly mean net hospital costs were $£ 34$ more per episode (5.3\% increase, $95 \% \mathrm{BCl}-11.6 \%$ to $25.5 \%$ ).

\section{Conclusions}

Despite annual increases in emergency department attendances, investment was associated with reduced waiting times for psychosocial assessment and more referrals to other agencies, with only a small increase in cost per episode.

\section{Keywords}

Self-harm; liaison psychiatry service; emergency department.

\section{Copyright and usage}

(c) The Author(s) 2020. This is an Open Access article, distributed under the terms of the Creative Commons Attribution licence (http://creativecommons.org/licenses/by/4.0/), which permits unrestricted re-use, distribution, and reproduction in any medium, provided the original work is properly cited.
Self-harm causes a significant international public health burden and is a key risk factor for deaths because of suicide. ${ }^{1-4}$ In England, around 200000 people present to emergency departments annually because of self-harm and more than $15 \%$ present again with repeated self-harm within a year. ${ }^{5,6}$ The direct and indirect costs associated with self-harm are considerable. ${ }^{7-11}$ Providing effective, evidence-based clinical care could reduce repeated self-harm attempts and suicide., ${ }^{2,12} \mathrm{UK}$ guidelines recommend that all patients presenting to healthcare because of self-harm should be offered a psychosocial assessment. ${ }^{13}$ The recommended assessment includes an evaluation of the factors leading to selfharm and a full mental health and social needs assessment. ${ }^{13}$ Despite this, the provision of care remains highly variable across UK hospitals. ${ }^{14-16}$

Liaison psychiatry services (LPS) provide care for patients presenting to emergency departments with mental health problems and those who have developed mental health problems while being treated in hospital. ${ }^{17}$ LPS provide psychiatric assessment and treatment to general hospital patients. These include those attending emergency departments as well as in-patients. A recent study of an expanded LPS in a large acute hospital in Birmingham indicated

* Joint first authors. that it led to a reduction in bed use with overall benefit to cost ratio of 4:1. ${ }^{17}$ In September 2014, Bristol Clinical Commissioning Group commissioned University Hospitals Bristol NHS Foundation Trust to expand the operating hours of the LPS at a large teaching hospital (Bristol Royal Infirmary, BRI). The working hours of the LPS service in BRI were extended from Monday to Friday $9.00-17.00 \mathrm{~h}$ ( $40 \mathrm{~h}$ per week) to 7 days a week $8.00-22.00 \mathrm{~h}$ (98 h per week). In previous work we have demonstrated that the LPS expansion led to several initial improvements in the management and outcomes of patients who have self-harmed. ${ }^{18}$ These included an increase in the proportion of self-harm attendances in the emergency department that received a psychosocial assessment, a reduction in median waiting time for an assessment and a reduction in the proportion of patients self-discharging without a psychosocial assessment. ${ }^{18}$ However, these results were based on a short-term comparison of 3 months before and 3 months after the LPS expansion. Current evidence on the long-term impact of LPS expansion remains limited. This study aims to assess the impact of the $£ 250000$ annual investment to expand the LPS in BRI on the outcomes of self-harm attendances, psychosocial assessments, repeat attendances and treatment costs for emergency department attendances for selfharm, over a 3-year period prior to and after service expansion. The findings will inform commissioners of the potential benefit of investing in LPS. 


\section{Method}

We used Bayesian structural time-series methods ${ }^{19,20}$ to evaluate the long-term impact of the expansion of LPS in Bristol, using anonymised data of patients presenting to the BRI emergency department following self-harm between 1 September 2011 and 30 September 2017. The study period was selected to cover a 3-year period before and after the LPS expansion beginning around September 2014.

\section{Data}

Data for all self-harm attendances at the emergency department were obtained from the Bristol Self-Harm Surveillance Register (BSHSR). The BSHSR is a database maintained in the emergency department of BRI that records detailed information on patients presenting to hospital for self-harm since $2010 .{ }^{21}$ Self-harm is defined in the BSHSR as intentional self-injury or self-poisoning irrespective of motivation or degree of suicidal intent. The BSHSR includes clinical and sociodemographic details of all hospitalpresentations for self-harm. Data collection is approved by the Central Bristol Research Ethics Committee.

Monthly summary data for each outcome were calculated to investigate the effect of the investment on the total number of episodes admitted to a hospital ward (observation, intensive therapy unit (ITU) or other general ward), the total number of self-harm episodes that received a psychosocial assessment, the total number of episodes self-discharging from the emergency department without a psychosocial assessment, the total number of patients who had a repeat emergency department attendance within 6 months of their index attendance (a patient's first selfharm attendance during the study period with no recorded selfharm attendance within the 6 months prior), the median waiting time (hours) from arriving in the emergency department to receiving a psychosocial assessment, the total number of referrals made to other agencies (including crisis, alcohol, self-harm, social services), and service costs. Psychosocial assessment costs were calculated according to the profession of the assessor, assuming a $90 \mathrm{~min}$ assessment using an average salary of a band 7 nurse (liaison nurse) or a weighted (80:20, reflecting the involvement of staff of different grades in assessments) average of junior (foundation year 1 and 2) and senior (registrar and consultant) doctor's salaries, plus overheads, oncosts and indirect time (see supplementary Table 1 available at https://doi.org/10.1192/bjo.2020.18 for cost breakdown). ${ }^{22}$ National Health Service (NHS) reference costs, ${ }^{23}$ stratified by whether the patient was subsequently admitted to hospital ( $£ 221.25)$ or not ( $£ 127.56)$, were used to estimate the costs of emergency department attendance. The average unit cost ( $£ 366.56)$ for a non-elective short-stay admission for 'observation and counselling' was used as a proxy for the daily cost of observational unit or other ward care and the average daily cost (£1250.69) of adult medical critical care patients was used to estimate costs for ITU days. Net hospital costs were calculated as the sum of the average psychosocial assessment, emergency department, observation and ITU costs each month.

Monthly averages were also obtained for covariates assumed to be unaffected by the investment, to be used in constructing a control time series predicting what would have happened to the outcomes if the investment had not happened: total number of attendances for self-harm, mean age of patients attending for self-harm and the proportion of patients attending for self-harm that were female.

\section{Statistical methods}

Structural time series models, ${ }^{19,20,24}$ in combination with Bayesian spike and slab regression and Bayesian model averaging, were used to estimate the impact of the expansion of the LPS in Bristol on each outcome. To describe what would have happened had the investment not been provided, a control time series (counterfactual) for each outcome was modelled using the measured pre-investment (1 September 2011 to 31 July 2014) data and the pre- and postinvestment time trends of the covariates described above. ${ }^{20,25,26}$ The implementation date of July 2014 was selected in order to avoid incorporating potential anticipatory effects shortly prior to the expansion of the LPS that came into effect in September 2014, such as the recruitment of liaison nurses. The post-investment period was 1 August 2014 to 30 September 2017.

Each outcome was modelled using a first-order autoregressive $(\mathrm{AR}(1))$ process, whereby the value of the outcome at each time point in the series is based on the value of the outcome at the immediately preceding time point taking into account random noise. A seasonal component was included to account for monthly fluctuations. See online supplementary material for model specifications. The impact of the investment was then estimated by comparing the observed time series of the measured outcomes during the post-investment period with the control time series.

The model assumes that any existing relationship between the time series of the covariates and the outcomes remained the same before and after the expansion of the LPS. This was confirmed by a sensitivity analysis (data not shown). Model fit was assessed using Geweke diagnostics, Raftery-Lewis diagnostic tests, mean absolute 1-step prediction errors, Durbin-Watson test, Ljung-Box test and autocorrelation plots.

Bayesian structural time series were constructed using the bsts package in $\mathrm{R},{ }^{27}$ and subsequently used as input for the Causal Impact $\mathrm{R}$ package. ${ }^{28}$ Results are presented as monthly averages over 3 years. Relative effects are presented as point estimates of the difference between the average observed and predicted values post-intervention as a percentage of the predicted value post-intervention, with Bayesian 95\% credible intervals (BCIs; the interval within which the true value falls with $95 \%$ probability, given the model and the data $\left.{ }^{29}\right)$. Posterior predictive $P$-values are calculated and interpreted as the posterior of the mean of classical $P$-values. ${ }^{30}$

\section{Results}

The temporal trends of the covariates (Fig. 1) indicate an overall increase in the mean number of attendances for self-harm (12 attendances) every month and the proportion of self-harm attendances made by females $(5.7 \%)$ in the post-investment period compared with the prior period. Mean age of all self-harm attendances in the post-investment period remained the same as that observed in the pre-investment period. The mean and range of each covariate and outcome in the time period before and after the investment are presented in supplementary Table 2 and graphically in Fig. 2. Comparing pre-investment and post-investment means: the mean number of self-harm attendances increased $13 \%$, the median waiting time from arrival in the emergency department to receiving a psychosocial assessment decreased from $11.6 \mathrm{~h}$ to $9.0 \mathrm{~h}$, the number of referrals made to other agencies increased from 47.1 to 97.6 , the number of episodes that received a psychosocial assessment increased from 53.0 to 65.8 and net hospital costs decreased from $£ 696.30$ to $£ 669.30$.

The results of the Bayesian modelling for each outcome are shown in Table 1 and graphically in Fig. 3 and supplementary Fig. 1. Comparing the counterfactual trends to the actual trends observed in the post-investment period, indicated that the median waiting time from arriving in the emergency department to receiving a psychosocial assessment was approximately $2 \mathrm{~h}$ shorter per month $(18.6 \%$ decrease, $95 \%$ BCI $-30.2 \%$ to $-2.8 \%)$, although this effect appears to be diminishing with time (Fig. 3(e)). The 
(a)

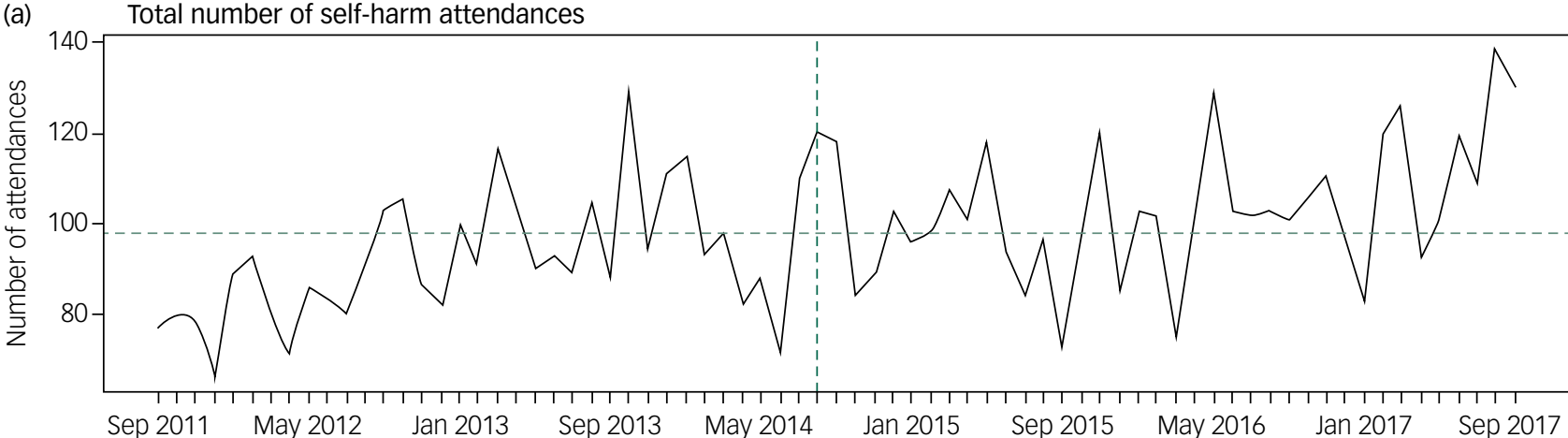

(b) Mean age of self-harm attendances
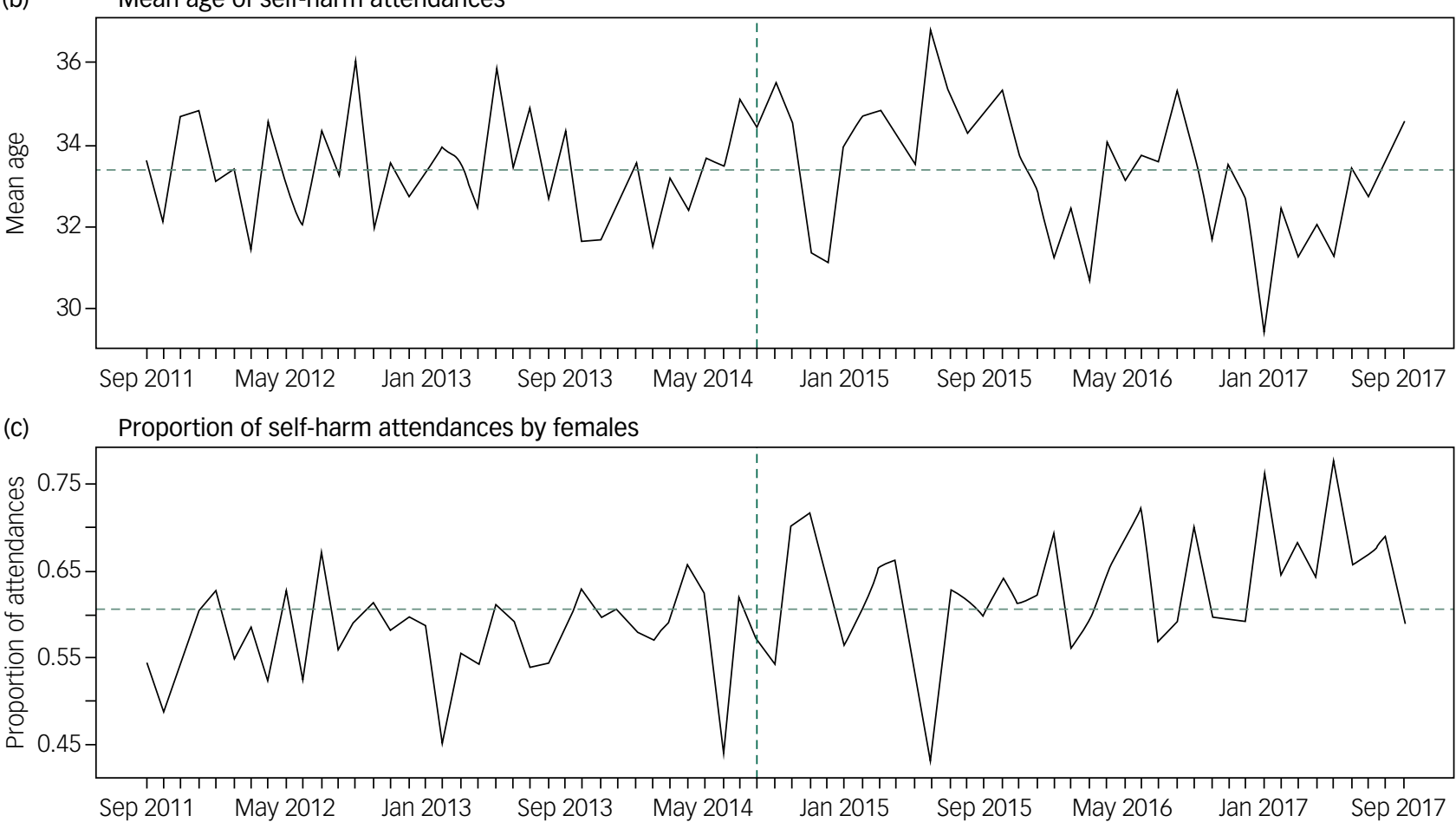

Fig. 1 Total number, mean age and proportion of female attendances of self-harm per month between September 2011 and September 2017.

mean cost of a psychosocial assessment increased by about $£ 18$ (10.3\% increase, $95 \%$ BCI $5.3 \%$ to $15.7 \%)$ and mean emergency department costs reduced by $£ 1.50$ per episode $(0.8 \%$ decrease, $95 \%$ BCI $-2.4 \%$ to $0.4 \%)$. There was little evidence of an effect on mean observation ward costs $(8.7 \%$ increase, $95 \% \mathrm{BCI}-12.0 \%$ to $35.3 \%)$, mean ITU costs $(4.6 \%$ decrease, $95 \%$ BCI $-120.5 \%$ to $129.2 \%)$ and mean net hospital costs (5.3\% increase, 95\% BCI $-11.6 \%$ to $25.5 \%)$.

Following the 2014 investment there were indications of an increase in the number of episodes that received a psychosocial assessment ( $11.7 \%$ increase, $95 \%$ BCI $-3.4 \%$ to $28.5 \%)$ and a decrease in the number of episodes self-discharging from the emergency department without a psychosocial assessment (7.7\% decrease, $95 \%$ BCI $-21.6 \%$ to $5.5 \%$ ), however, the evidence is inconclusive.

There were approximately 45 referrals more per month to other agencies $(86.1 \%$ increase, $95 \%$ BCI $60.6 \%$ to $110.9 \%)$ and there was little evidence that median time to first repeat attendance had changed (12 days longer per month, 28.6\% increase, 95\% BCI $-32.6 \%$ to $104.9 \%)$.

All models produced stable estimates and demonstrated acceptable convergence for the majority of variables after 100000 Markov chain Monte Carlo samples. Comparison of the observed and modelled data during the pre-investment period indicated that the observed time series were accurately modelled, with a mean absolute 1 -step prediction error less than $10 \%$ of the pre-investment outcome mean for the majority of outcomes (64\%) and between $10 \%$ and $30 \%$ for the remainder. As the assumption that there is no change in correlations between the outcomes and covariates post-investment is correct then the counterfactual time series is expected to be of similar accuracy.

\section{Discussion}

\section{Main findings}

Following the 2014 investment, there was a decrease in the waiting time from emergency department arrival to receiving a psychosocial assessment, a considerable increase in the number of referrals to other agencies and an increase in the absolute number of psychosocial assessments. Evaluation of costs showed an increase in average cost of a psychosocial assessment, however, there was little evidence of an overall increase in mean net hospital costs. 


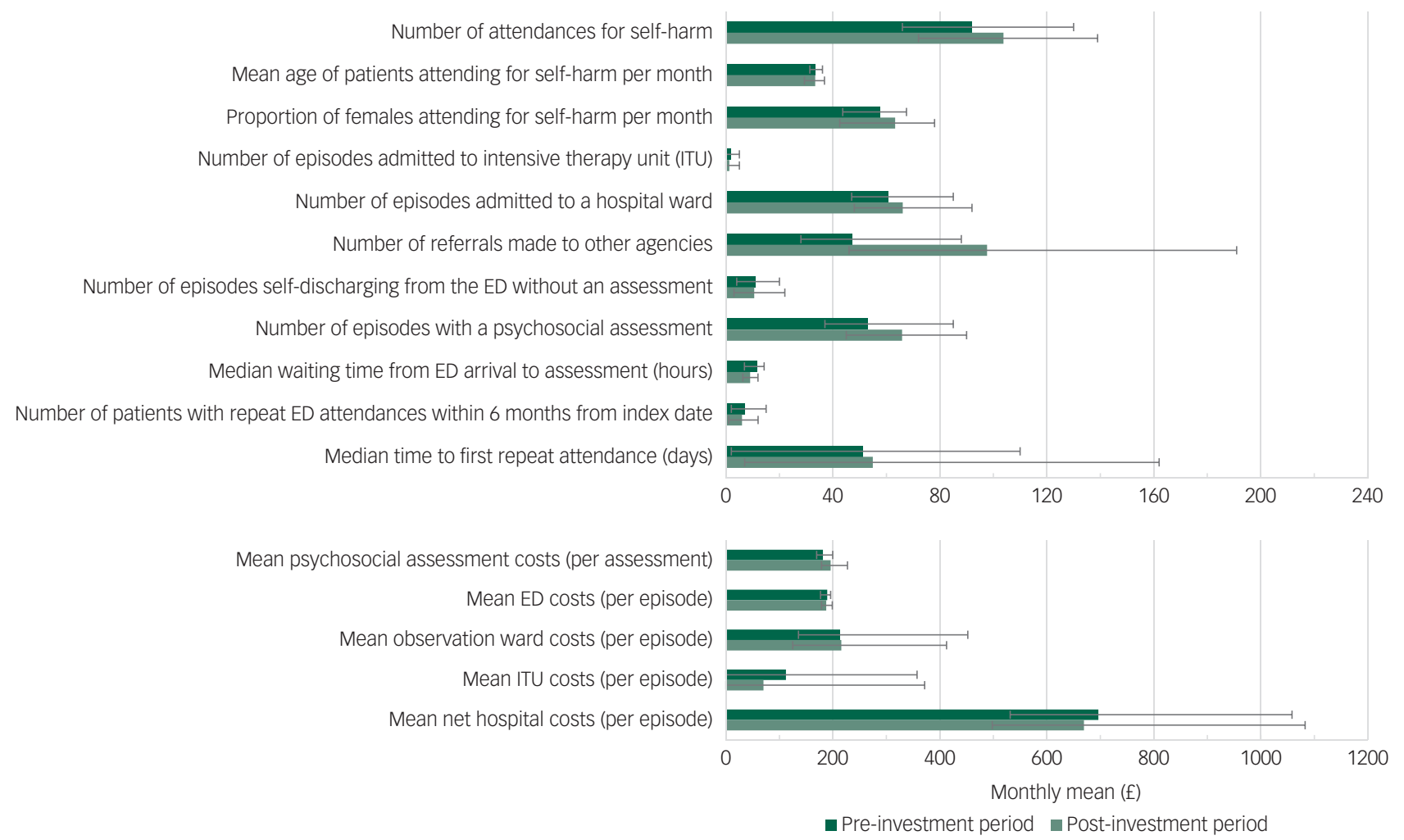

Fig. 2 Monthly mean values of covariates and outcomes in the pre-investment period (dark green) and post-investment period (light green).

\section{Comparison with other studies}

UK clinical guidelines recommend psychosocial assessment for all patients presenting with self-harm. ${ }^{13}$ The findings of this evaluation suggest that the short-term decrease in median waiting time from arrival in emergency department to psychosocial assessment identified by Opmeer et $a l^{18}$ has persisted longer term, with a median $2 \mathrm{~h}$ reduction in waiting times. Short-term increases in referrals to the crisis or other community teams also appear to have continued, with an overall increase in referrals made to other agencies identified long term. In contrast, evidence for a reduction in the number of episodes self-discharging without an assessment was less clear than in the initial evaluation. ${ }^{18}$ There was an indication of an increase in the number of episodes that received a psychosocial assessment, however, this finding was also less clear than in the initial evaluation. Neither evaluation identified an effect on the number of patients with repeat self-harm attendances or median time to first repeat following the investment.

\begin{tabular}{|c|c|c|c|}
\hline Outcome & $\begin{array}{l}\text { Relative average } \\
\text { effect, \% }(95 \% \mathrm{BCl})\end{array}$ & $\begin{array}{l}\text { Absolute average } \\
\text { monthly effect of } \\
\text { the investment }\end{array}$ & $\begin{array}{c}\text { Posterior } \\
\text { predictive } P\end{array}$ \\
\hline \multicolumn{4}{|l|}{ Episode outcomes } \\
\hline Number of episodes admitted to intensive therapy unit & $-28.3(-49.5$ to -6.8$)$ & -0.5 episodes & 0.008 \\
\hline Number of episodes admitted to a hospital ward & $0.1(-4.3$ to 3.6$)$ & +0.1 episodes & 0.449 \\
\hline Number of referrals made to other agencies & 86.1 (60.6 to 110.9$)$ & +45.2 referrals & $<0.001$ \\
\hline $\begin{array}{l}\text { Number of episodes self-discharging from the emergency department } \\
\text { without an assessment }\end{array}$ & -7.7 (-21.6 to 5.5$)$ & -0.9 episodes & 0.102 \\
\hline \multicolumn{4}{|l|}{ Psychosocial assessments } \\
\hline Number of episodes with a psychosocial assessment & $11.7(-3.4$ to 28.5$)$ & +6.9 episodes & 0.043 \\
\hline Median waiting time from emergency department arrival to assessment, $\mathrm{h}$ & $-18.6(-30.2$ to -2.8$)$ & $-2.1 \mathrm{~h}$ & 0.014 \\
\hline \multicolumn{4}{|l|}{ Repeat attendances } \\
\hline $\begin{array}{l}\text { Number of patients with repeat emergency department attendances within } \\
6 \text { months from index date }\end{array}$ & -11.2 (-39.6 to 27.3) & -0.8 patients & 0.198 \\
\hline Median time to first repeat attendance, days & $28.6(-32.6$ to 104.9$)$ & +12.2 days & 0.186 \\
\hline \multicolumn{4}{|l|}{ Cost outcomes } \\
\hline Mean psychosocial assessment costs & 10.3 (5.3 to 15.7$)$ & $+£ 18.3$ per assessment & $<0.001$ \\
\hline Mean emergency department costs & $-0.8(-2.4$ to 0.4$)$ & -£1.5 per episode & 0.092 \\
\hline Mean observation ward costs & $8.7(-12.0$ to 35.3$)$ & +£17.3 per episode & 0.183 \\
\hline Mean intensive therapy unit costs & $-4.6(-120.5$ to 129.2$)$ & -£3.4 per episode & 0.439 \\
\hline Mean net hospital costs & $5.3(-11.6$ to 25.5$)$ & +£33.6 per episode & 0.261 \\
\hline
\end{tabular}


(a)

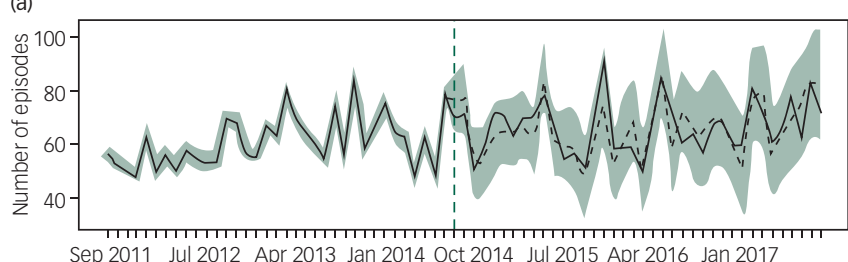

(c)
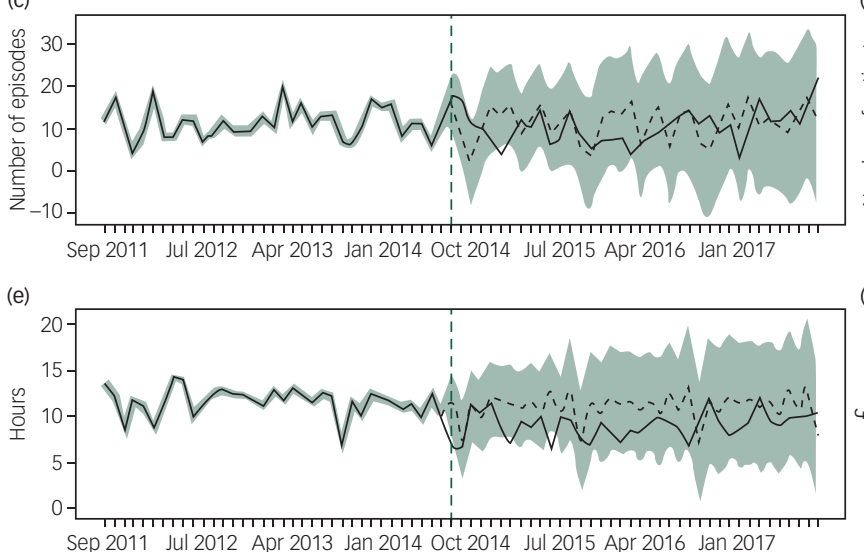
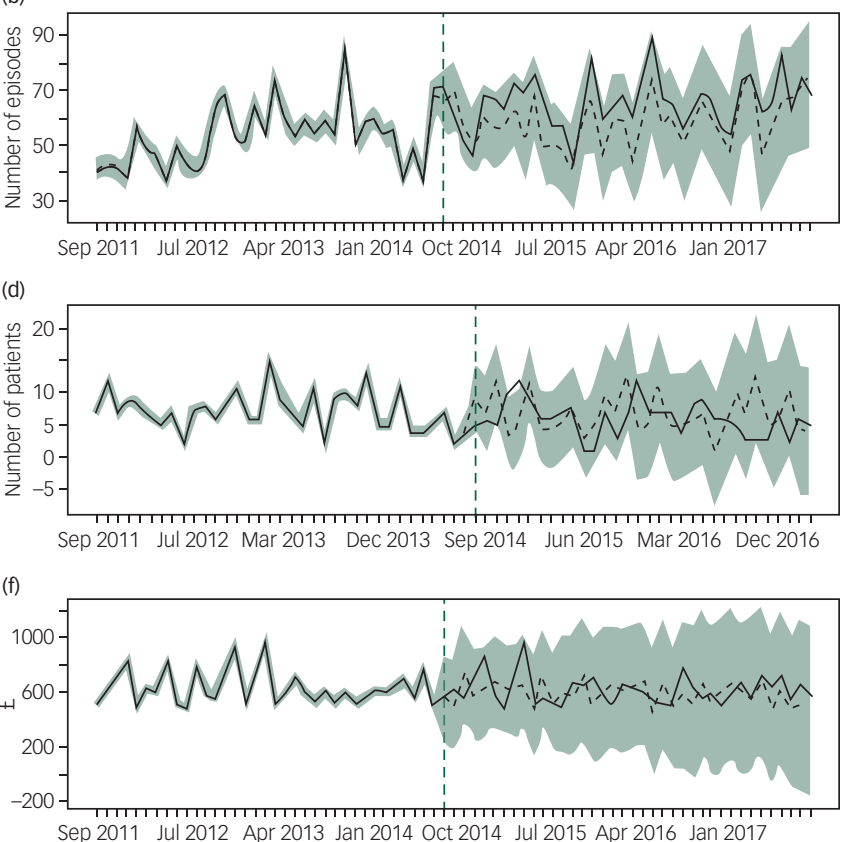

\section{Fig. 3 Observed (solid line) and modelled (dashed line) time series.}

Time series for (a) the number of episodes admitted to a hospital ward; (b) the number of episodes with a psychosocial assessment; (c) the number of episodes self-discharging from the emergency department without an assessment; (d) the number of patients with repeat emergency department attendances within 6 months from index attendance; (e) the median waiting time from emergency department arrival to assessment (hours); (f) mean net hospital costs. Shaded areas correspond to $95 \%$ credible intervals.

Economic evaluation of the RAID (rapid assessment interface and discharge) LPS at a large acute hospital in Birmingham reported cost savings based on reduced length of stay, avoided admissions to general wards and reduced rates of readmission, with the majority of savings as a result of shorter lengths of stay among older patients (>65 years). ${ }^{17}$ Less than $28 \%$ of referrals to RAID were for selfharm, however, whereas the current evaluation focused solely on LPS activities for patients who have self-harmed who comprise only $40 \%$ of the liaison team workload.

\section{Interpretation of results}

Despite the annual increase in the number of attendances for self-harm the number (and proportion) of episodes receiving a psychosocial assessment increased and the number of episodes self-discharging without an assessment fell, indicating the resilience of the LPS in face of mounting pressures. Patient's risk is assessed at triage using a risk matrix, with higher-risk patients prioritised for psychosocial assessment. Further research is required to understand the reasons underlying instances of non-assessment of patients referred for psychosocial assessment, but may include selfdischarge, patient's refusal, low-risk score, under existing psychiatric care. ${ }^{31}$ The diminishing effect of the investment on waiting time from emergency department arrival to receiving psychosocial assessment demonstrates a continuing need for investment in order to meet service demands in light of the annual increases in attendances. The small (5\%) increase in net costs identified in this evaluation is most likely a reflection of the increase in the proportion of psychosocial assessments carried out by liaison nurses that are associated with a higher cost than assessments carried out by a doctor (often trainee doctors). The inferred increase in referrals to other agencies is possibly a reflection of improved risk assessment and patient management following the investment, potentially as a result of the increased capacity of liaison nurses, who are generally more experienced than trainee doctors in the emergency department and likely to have a more detailed knowledge of potential local referral agencies. The increase in psychosocial assessment costs may be offset by such improvements in the management of patients who self-harm and referral to appropriate services if these result in improved patient outcomes.

\section{Strengths}

The methods used in the current study not only enabled the evaluation of the effects of the intervention over a longer, 3-year period of time than the previous evaluation by Opmeer et al, ${ }^{18}$ but was also able to account for secular and seasonal trends. In addition, in the absence of randomised controls, the Bayesian structural time series method enabled the construction of a synthetic control to act as a comparison with the observed data. Finally, Bayesian model averaging minimises issues of arbitrary and incorrect covariate selection and of overfitting. ${ }^{20}$

Compared with previous studies based on select groups of patients (such as those who had taken an overdose) $)^{32}$ or on small numbers of patients, ${ }^{11}$ the data used in this analysis was taken from an unselected series of consecutive presentations of selfharm, including those that were admitted to hospital as well as those with emergency department attendance that did not result in admission.

\section{Limitations}

An important limitation is that unobserved variables might have improved the counterfactual estimation, thereby reducing the potential of bias. Through the use of Bayesian model averaging in combination with spike and slab priors inclusions of the covariates were weighted to avoid overfitting, but we cannot exclude that other variables, not a priori selected, could have improved model fit and may have further improved forecasting accuracy. 
Despite several methodological advantages of the use of this Bayesian framework, a limitation is that it requires more data than non-Bayesian methods to obtain comparable forecasting precision and to adequately model the counterfactual. The relatively wide credible intervals around important outcomes, such as reattendance for self-harm, indicate that a larger study would be required to provide stronger evidence.

As noted by Opmeer et al, ${ }^{18}$ of all LPS referrals, psychosocial assessment of people attending emergency department for selfharm comprises only $40 \%$. Although the expansion of the LPS operating hours is likely to result in a better service for all patients with psychiatric morbidity, neither the short-term nor long-term evaluations measured the impact of other (non-self-harm related) activities of the liaison psychiatry team at BRI. Furthermore, we were unable to assess the full economic impact of the LPS on NHS costs as the BSHSR does not record details of subsequent primary and community care services. The increase in referrals to other agencies could plausibly either increase or decrease the long-term costs of care for patients who have self-harmed.

Joni Jackson (D), BSC, MSC, The National Institute for Health Research Applied Research Collaboration West (NIHR ARC West), University Hospitals Bristol NHS Foundation Trust; and Population Health Sciences, Bristol Medical School, University of Bristol, UK; Manjula D. Nugawela, BSC, MSC, PhD, The National Institute for Health Research Applied Research Collaboration West (NIHR ARC West), University Hospitals Bristol NHS Foundation Trust; and Population Health Sciences, Bristol Medical School, University of Bristol, UK; Frank De Vocht, BSC, MSC, PhD, Bristol Medical School, University of Bristol UK; Paul Moran, MB, BS, MSC, MD, FRCPsych, Centre for Academic Mental Health, Population Health Sciences, Bristol Medical School, University of Bristol, UK: William Hollingworth, BSC, MSC, PhD, Population Health Sciences, Bristol Medical School, University of Bristol, UK; Duleeka Knipe (iD), PhD, Population Health sciences, Bristol Medical School, University of Bristol, UK; Nik Munien, RN(M), BA University Hospitals Bristol NHS Foundation Trust, UK; David Gunnell, MB, ChB, PhD, FFPH DSC, Population Health Sciences, Bristol Medical School, University of Bristol, UK; Maria Theresa Redaniel, BSPH, MSC, PhD, The National Institute for Health Research Applied Research Collaboration West (NIHR ARC West), University Hospitals Bristol NHS Foundation Trust; and Population Health Sciences, Bristol Medical School, University of Bristol, UK

Correspondence: Joni Jackson. Email: joni.jackson@bristol.ac.uk

First received 27 Sep 2019, final revision 28 Feb 2020, accepted 9 Mar 2020

\section{Supplementary material}

Supplementary material is available online at https://doi.org/10.1192/bjo.2020.18.

\section{Data availability}

Data associated with this manuscript is accessible only to the research team and is not publicly available

\section{Acknowledgements}

Authors would like to acknowledge the liaison psychiatry service clinical staff, the steering group for the Bristol self-harm register and the Improving Care in Self-Harm Health Integration Team (STITCH HIT).

\section{Author contributions}

M.T.R., D.G., P.M. and D.K. conceived and formulated the research question and designed the study. J.J. and M.D.N. analysed the data, supervised by M.T.R. and F.D.V. W.H. provided health economic expertise and P.M. and N.M. clinical expertise. All authors discussed the results and contributed to the final manuscript.

\section{Funding}

This research was funded by the National Institute for Health Research Applied Research Collaboration West (NIHR ARC West). The views expressed in this article are those of the author(s) and not necessarily those of the NIHR or the Department of Health and Social Care.

\section{Declaration of interest}

None.

ICMJE forms are in the supplementary material, available online at https://doi.org/10.1192/bjo. 2020.18.

\section{References}

1 Murray CJ, Vos T, Lozano R, Naghavi M, Flaxman AD, Michaud C, et al. Disabilityadjusted life years (DALYS) for 291 diseases and injuries in 21 regions, 19902010: a systematic analysis for the Global Burden of Disease Study 2010. Lancet 2012; 380: 2197-223.

2 World Health Organization. Preventing Suicide: A Global Imperative. WHO, 2014 (http://apps.who.int/iris/bitstream/10665/131056/1/9789241564779_eng.pdf).

3 Hawton K, Zahl D, Weatherall R. Suicide following deliberate self-harm: longterm follow-up of patients who presented to a general hospital. $\mathrm{Br} J$ Psychiatry 2003; 182: 537-42.

4 Hawton $\mathrm{K}$, Bergen $\mathrm{H}$, Cooper J, Turnbull P, Waters K, Ness J, et al. Suicide following self-harm: findings from the Multicentre Study of self-harm in England, 2000-2012. J Affect Disord 2015; 175: 147-51.

5 Carroll R, Metcalfe C, Gunnell D. Hospital presenting self-harm and risk of fatal and non-fatal repetition: systematic review and meta-analysis. PLOS ONE 2014; 9: e89944.

6 Carroll R, Metcalfe C, Gunnell D. Hospital management of self-harm patients and risk of repetition: systematic review and meta-analysis. J Affect Disord 2014: 168: 476-83.

7 Sinclair JM, Gray A, Rivero-Arias O, Saunders KE, Hawton K. Healthcare and social services resource use and costs of self-harm patients. SOC Psychiatry Psychiatr Epidemiol 2011; 46: 263-71.

8 Goldman-Mellor SJ, Caspi A, Harrington H, Hogan S, Nada-Raja S, Poulton R, et al. Suicide attempt in young people: a signal for long-term health care and social needs. JAMA Psychiatry 2014; 71: 119-27.

9 Shepard DS, Gurewich D, Lwin AK, Reed GA, Jr, Silverman MM. Suicide and suicidal attempts in the united states: costs and policy implications. Suicide Life Threat Behav 2016; 46: 352-62.

10 Kapur N, House A, Dodgson K, May C, Marshall S, Tomenson B, et al. Management and costs of deliberate self-poisoning in the general hospital: a multi-centre study. J Ment Health 2002; 11: 223-30.

11 Sgobin SM, Traballi AL, Botega NJ, Coelho OR. Direct and indirect cost of attempted suicide in a general hospital: cost-of-illness study. Sao Paulo Med J 2015; 133: 218-26.

12 Carroll R, Metcalfe C, Steeg S, Davies NM, Cooper J, Kapur N, et al. Psychosocial assessment of self-harm patients and risk of repeat presentation: an instrumental variable analysis using time of hospital presentation. PLOS ONE 2016; 11: e0149713.

13 National Collaborating Centre for Mental Health. Self-Harm: The Short-Term Physical and Psychological Management and Secondary Prevention of SelfHarm in Primary and Secondary Care. National Institute for Health and Clinical Excellence: Guidance. British Psychological Society and The Royal College of Psychiatrists, 2004.

14 Cooper J, Steeg S, Bennewith O, Lowe M, Gunnell D, House A, et al. Are hospita services for self-harm getting better? An observational study examining management, service provision and temporal trends in England. BMJ Open 2013; 3: e003444.

15 Kapur N, House A, Creed F, Feldman E, Friedman T, Guthrie E. Management of deliberate self poisoning in adults in four teaching hospitals: descriptive study. BMJ 1998; 316: 831-2.

16 Bennewith O, Gunnell D, Peters TJ, Hawton K, House A. Variations in the hospital management of self harm in adults in England: observational study. BMJ 2004; 328: 1108-9.

17 Parsonage M, Fossey M. Economic Evaluation of a Liaison Psychiatry Service. Centre of Mental Health, 2011 (https://www.centreformentalhealth.org.uk/ Handlers/Download.ashx?IDMF=d6fa08e0-3c6a-46d4-8c07-93f1d44955e8).

18 Opmeer BC, Hollingworth W, Marques EMR, Margelyte R, Gunnell D. Extending the liaison psychiatry service in a large hospital in the UK: a before and after evaluation of the economic impact and patient care following ED attendances for self-harm. BMJ Open 2017; 7: e016906.

19 Brodersen KH, Gallusser F, Koehler J, Remy N, Scott SL. Inferring causal impact using Bayesian structural time-series models. Ann Appl Stat 2015; 9: 247-74.

20 Scott SL, Varian HR. Predicting the present with Bayesian structural time series. Int J Math Model Numer Optim 2014; 5: 4-23.

21 Bristol Self Harm Surveillance Register. Annual Report - 2016. Bristol Self Harm Surveillance Register, 2017 (http://www.bristolhealthpartners.org.uk/ uploads/documents/2017-08-23/1503503311-bshsr-annualreport-april-2017. pdf).

22 Curtis LA, Burns A. Unit Costs of Health and Social Care 2018. University of Kent, 2018.

23 NHS Improvement. Reference Cost Collection: National Schedule of Reference Cost, 2016-17. NHS Trusts and NHS Foundation Trusts Improvement, 2018 (https://improvement.nhs.uk/resources/reference-costs/). 
24 de Vocht F, Tilling K, Pliakas T, Angus C, Egan M, Brennan A, et al. The intervention effect of local alcohol licensing policies on hospital admission and crime: a natural experiment using a novel Bayesian synthetic time-series method. $J$ Epidemiol Community Health 2017; 71: 912.

25 Abadie A, Diamond A, Hainmueller J. Synthetic control methods for comparative case studies: estimating the effect of california's tobacco control program. J Am Stat Assoc 2010; 105: 493-505.

26 Abadie A, Gardeazabal J. The economic costs of conflict: a case study of the Basque country. Am Econ Rev 2003; 93: 113-32.

27 Scott SL. Package 'bsts' 2016. CRAN, 2019 (https://cran.r-project.org/web/ packages/bsts/bsts.pdf)

28 Brodersen $\mathrm{KH}$. CausalImpact. An R Package for Causal Inference Using Bayesian Structural Time-Series Models. Google, 2014-2016.
29 Kruschke JK, Liddell TM. The Bayesian new statistics: hypothesis testing, estimation, meta-analysis, and power analysis from a Bayesian perspective. Psychon Bull Rev 2018; 25: 178-206.

30 Meng X-L. Posterior predictive P-values. Ann Statist 1994; 22: 1142-60.

31 Bennewith O, Peters TJ, Hawton K, House A, Gunnell D. Factors associated with the non-assessment of self-harm patients attending an accident and emergency department: results of a national study. J Affect Disord 2005; 89: 91-7.

32 Kapur N, House A, Dodgson K, May C, Creed F. Effect of general hospital management on repeat episodes of deliberate self poisoning: cohort study. BMJ 2002; 325: 866-7. 